\title{
Galilean invariance and homogeneous anisotropic randomly stirred flows
}

\author{
Arjun Berera 1 , : 6 and David Hochberg 2 , 周 \\ ${ }^{1}$ School of Physics, University of Edinburgh, Edinburgh, EH9 3JZ, U.K. \\ ${ }^{2}$ Centro de Astrobiología (CSIC-INTA), Ctra. Ajalvir Km. 4, 28850 Torrejón de Ardoz, Madrid, Spain
}

(Dated: March 15, 2018)

\begin{abstract}
The Ward-Takahashi (WT) identities for incompressible flow implied by Galilean invariance are derived for the randomly forced Navier-Stokes equation (NSE), in which both the mean and fluctuating velocity components are explicitly present. The consequences of Galilean invariance for the vertex renormalization are drawn from this identity.
\end{abstract}

PACS numbers: 47.27.Ak, 11.10.Gh

The transformation properties of the Navier-Stokes equation (NSE) play an important part in both the physics of turbulence as well as in practical aspects of turbulence modelling [1]. Among the invariance properties or symmetries of the NSE, Galilean invariance is perhaps the most frequently cited in theoretical approaches to turbulence [2, 3, 4, 5, 6].

For fluctuating flows, a decomposition of the instantaneous fluid velocity into a mean plus a component fluctuating relative to the mean (the Reynolds decomposition) shows that the mean and fluctuating velocities respond to a Galilean transformation in different ways. This would seem to be the most general physical significance one could deduce by studying the behavior of the NSE under Galilean transformations. On the other hand, because the Galilean transformation is a symmetry of the NSE, it leads to nontrivial and exact identities among various correlation functions. These so-called Ward-Takahashi (WT) identities make two types of important statements: (a) In quantum field theory (QFT), they relate formally divergent parts of different correlation functions. WardTakahashi identities reduce the number of counterterms or renormalization constants; and $(b)$ in QFT as well as in field theoretic approaches to condensed matter problems, they relate different physical quantities. For example, in hydrodynamics they relate the eddy viscosity and the numerical prefactor multiplying the nonlinear convective term (the vertex). Thus, the deeper physical significance of the Galilean transformation is that it constrains the values of the physical parameters appearing in the NSE, and in particular, it leads to the oft cited non-renormalization of the vertex of the NSE in the large-scale flow regime [2].

Recently however it was claimed that Galilean invariance does not at all constrain the vertex renormalization 7], thus putting into serious question the widely cited key early papers in turbulence research [2, 3]. In 7], a distinction is drawn between the NSE for the instantaneous velocity field $V_{i}$ and the NSE for the fluctuating velocity $u_{i}$ in the comoving frame of the mean constant velocity, for

*Electronic address: ab@ph.ed.ac.uk

${ }^{\dagger}$ Electronic address: hochberg@laeff.esa.es spatially homogeneous flow. These velocities are defined through the Reynolds decomposition of the velocity into its mean plus fluctuations: $V_{i}(\mathbf{x}, t)=\left\langle V_{i}(\mathbf{x}, t)\right\rangle+u_{i}(\mathbf{x}, t)$, where the angular brackets denote the ensemble average 1]. The essential observation made in 7] is that Galilean invariance of the equation for the fluctuating velocity $u_{i}$ is established by transforming the mean velocity only. No previous field theoretic derivations of the vertex non-renormalization result have distinguished between the mean and fluctuating velocity components, since they treat the instantaneous velocity field at zero mean velocity, in other words, the case of a homogeneous and isotropic flow. In order to address specifically the concern in [7], in which the mean velocity plays a distinguished role, one should examine carefully the derivation and physical consequence of the pertinent WT identities, keeping explicit the decomposition of the instantaneous velocity field into its fluctuating and generally nonzero mean components. In short, one needs to treat the case of a homogeneous but anisotropic fluid. The purpose of this Brief Report is to carry this out in some detail, paying careful attention to the rather different ways the mean and fluctuating velocities are affected by a Galilean transformation. The issue of vertex renormalization will be addressed within this framework.

We start with the Reynolds decomposition to express the NSE in terms of the constant mean velocity and the fluctuation about the mean. So we take $V_{i}=K_{i}+u_{i}$, where $K_{i}=\left\langle V_{i}\right\rangle$ is the constant mean velocity and $u_{i}$ is the fluctuation about the mean. By definition, $\left\langle u_{i}\right\rangle=$ 0 . As demonstrated in [7], the equation of motion for a constant mean velocity $K_{i}$ vanishes identically, resulting in the NSE for the fluctuating velocity $u_{i}$. To this we add a stirring force $f_{i}$ and express the equation in solenoidal form:

$$
\frac{\partial u_{i}}{\partial t}+K_{j} \frac{\partial u_{i}}{\partial x_{j}}+\lambda_{0} P_{i j}(\nabla) \frac{\partial\left(u_{l} u_{j}\right)}{\partial x_{l}}=\nu_{0} \nabla^{2} u_{i}+f_{i},
$$

where the projection operator is $P_{i j}(\nabla)=\left(\delta_{i j}-\right.$ $\left.\nabla_{i} \frac{1}{\nabla^{2}} \nabla_{j}\right) . f_{i}$ is Gaussian with zero mean $\left\langle f_{i}\right\rangle=0$ and time and space translation invariant: $\left\langle f_{i}(\mathbf{x}, t) f_{j}(\mathbf{y}, \tau)\right\rangle=$ $D_{i j}(\mathbf{x}-\mathbf{y}) \delta(t-\tau)$. The viscosity is written with subscript zero, $\nu_{0}$, to remind us that this is the bare (unrenormalized) molecular viscosity that appears in the fluid equa- 
tion of motion. We provide the nonlinear term in Eq. (11) with a parameter $\lambda_{0}=1$. This is a well-known convenient bookkeeping device used in perturbation theory, in which typically, solutions of Eq. (1) are developed formally in powers of $\lambda_{0}$.

As we are interested in the correlation functions implied by Eq. (11), we make use of dynamic functional methods 8, 9, 10, 11]. The generating functional for the correlation functions associated with Eq. (1) is given by

$$
\begin{aligned}
Z[\mathbf{J}, \boldsymbol{\Sigma}]_{K} & =\int[\mathcal{D} \mathbf{u}][\mathcal{D} \sigma] \exp \left\{-S[\mathbf{u}, \sigma]_{K}\right. \\
& \left.+\int d \mathbf{x} d t(\mathbf{J} \cdot \mathbf{u}+\mathbf{J} \cdot \mathbf{K}+\boldsymbol{\Sigma} \cdot \sigma)\right\},
\end{aligned}
$$

where the action $S$ is

$$
\begin{aligned}
& S[\mathbf{u}, \sigma]_{K}=\int d \mathbf{x} d t\left(\frac{1}{2} \int d \mathbf{y} \sigma_{i}(\mathbf{x}, t) D_{i j}(\mathbf{x}-\mathbf{y}) \sigma_{j}(\mathbf{y}, t)\right. \\
& \left.-i \sigma_{k}\left(\frac{\partial u_{k}}{\partial t}+K_{j} \frac{\partial u_{k}}{\partial x_{j}}+\lambda_{0} P_{k j}(\nabla) \frac{\partial\left(u_{l} u_{j}\right)}{\partial x_{l}}-\nu_{0} \nabla^{2} u_{k}\right)\right)
\end{aligned}
$$

The field $\sigma$ is conjugate to the velocity $u_{i}$, and is the ana$\log$ of the noncommuting operator introduced by Martin, Siggia and Rose (MSR) in their operator formalism of classical statistical dynamics 12 . J and $\boldsymbol{\Sigma}$ are arbitrary space and time dependent source functions for $\mathbf{V}=\mathbf{u}+\mathbf{K}$ and $\sigma$, respectively. It is important to note that both the mean and fluctuating velocities couple to the same external source. A straightforward and convincing way to see that this must be so is to start from the forced NSE for the instantaneous velocity $V_{i}$ and set up its corresponding action and dynamic functional. Couple $V_{i}$ to a source term $J_{i}$. Insert the Reynolds decomposition $V_{i}=K_{i}+u_{i}$ into the measure, action and into the source terms. The incompressibility of the flow implies the fluctuations are themselves incompressible: $\nabla_{i} V_{i}=0 \Rightarrow \nabla_{i} u_{i}=0$. Then Eqs. (2) and (3) follow immediately from the dynamic functional and action for the instantaneous velocity.

We now subject the generating functional $Z$ to a Galilean transformation. Consider a second primed frame moving with constant velocity $\mathbf{c}$ with respect to the unprimed frame. Then the relations between time and coordinates of an event in the unprimed and primed frames are $t=t^{\prime}$ and $\mathbf{x}=\mathbf{c} t+\mathbf{x}^{\prime}$ while the velocities and conjugate field transform as $K_{i}=c_{i}+K_{i}^{\prime}, u_{i}(\mathbf{x}, t)=$ $u_{i}^{\prime}\left(\mathbf{x}^{\prime}, t^{\prime}\right)$ and $\sigma_{i}(\mathbf{x}, t)=\sigma_{i}^{\prime}\left(\mathbf{x}^{\prime}, t^{\prime}\right)$. Note that the mean and fluctuating velocities are affected in different ways. The conjugate field $\sigma$ has dimensions of acceleration and so transforms as indicated above. Using the time and coordinate transformations as well as these rules, it is easy to verify that $S\left[\mathbf{u}^{\prime}, \sigma^{\prime}\right]_{K^{\prime}}=S[\mathbf{u}, \sigma]_{K}$ and the functional integral measures are invariant: $\left[\mathcal{D} \mathbf{u}^{\prime}\right]=[\mathcal{D} \mathbf{u}],\left[\mathcal{D} \sigma^{\prime}\right]=[\mathcal{D} \sigma]$.

To obtain the Ward-Takahashi identity, consider an infinitesimal Galilean transformation and Taylor expand the fluctuating velocity out to first order in the relative velocity $\delta \mathbf{c}$ as follows:

$$
\mathbf{u}(\mathbf{x}+\delta \mathbf{c} t, t)=\mathbf{u}(\mathbf{x}, t)+t(\delta \mathbf{c} \cdot \nabla) \mathbf{u}(\mathbf{x}, t),
$$

with an analogous expansion for $\sigma$. This implies that the functional $Z$ in Eq. (2) transforms as $Z \rightarrow Z+\delta Z$, where

$$
\delta Z=\left\langle\left(\int t J_{l}(\delta \mathbf{c} \cdot \nabla) u_{l}+t \Sigma_{l}(\delta \mathbf{c} \cdot \nabla) \sigma_{l}-\mathbf{J} \cdot \delta \mathbf{c}\right)\right\rangle_{\bullet}=0 .
$$

The angular brackets with bullet denote the average taken over the turbulent ensemble in the presence of the source terms. As Eq. (5) holds for all $\delta \mathbf{c}$, it follows that $Z$ satisfies the functional differential equation (the integral is over $\mathbf{x}, t$ )

$$
\left(\int t J_{l} \nabla_{j} \frac{\delta}{\delta J_{l}}+t \Sigma_{l} \nabla_{j} \frac{\delta}{\delta \Sigma_{l}}-J_{j}\right) Z[\mathbf{J}, \boldsymbol{\Sigma}]_{K}=0 .
$$

This makes use of the fact that the mean velocity $K_{l}$ is constant in homogeneous turbulence, hence $\nabla_{j}\left\langle K_{l}\right\rangle_{\bullet}=$ $\nabla_{j} K_{l}\langle 1\rangle_{\bullet}=K_{l} \nabla_{j}\langle 1\rangle_{\bullet}=0$, and so $\nabla_{j} \frac{\delta}{\delta J_{l}} Z[\mathbf{J}, \mathbf{\Sigma}]_{K}=$ $\nabla_{j}\left\langle u_{l}+K_{l}\right\rangle_{\bullet}=\nabla_{j}\left\langle u_{l}\right\rangle_{\bullet}+\nabla_{j}\left\langle K_{l}\right\rangle_{\bullet}=\nabla_{j}\left\langle u_{l}\right\rangle_{\bullet}$. This identity Eq. (6) for the functional $Z$ can be used to obtain exact relationships among the correlation functions associated with the random velocity field in its Reynolds decomposed form. Of course, $Z$ generates both the disconnected and connected correlation functions. For the purposes of renormalization, it is customary to work instead with the connected proper vertices, also known as the one-particle irreducible (1PI) Green functions 13. It is important to point out that at lowest order, or tree-level, the connected proper vertex functions coincide with the vertices of the original (bare) action $S$ (3). To obtain the generating functional for 1PI vertices, we follow standard practice and introduce the generating functional of the connected Green functions $W=\ln Z$, and then carry out a Legendre transform 13. $\Gamma\left[\langle\mathbf{V}\rangle_{\bullet},\langle\sigma\rangle_{\bullet}\right]=-W[\mathbf{J}, \boldsymbol{\Sigma}]+\int \mathbf{J} \cdot\langle\mathbf{V}\rangle_{\bullet}+\boldsymbol{\Sigma} \cdot\langle\sigma\rangle_{\bullet}$, where

$$
\left\langle V_{k}\right\rangle_{\bullet}=\frac{\delta W}{\delta J_{k}},\left\langle\sigma_{k}\right\rangle_{\bullet}=\frac{\delta W}{\delta \Sigma_{k}}, \frac{\delta \Gamma}{\delta\left\langle V_{k}\right\rangle_{\bullet}}=J_{k}, \frac{\delta \Gamma}{\delta\left\langle\sigma_{k}\right\rangle_{\bullet}}=\Sigma_{k} .
$$

Note of course that $\left\langle V_{k}\right\rangle_{\bullet}=\left\langle u_{k}\right\rangle_{\bullet}+K_{k}$. From the definitions of $W$ and $\Gamma$ and using Eq. (7), we obtain immediately the Ward-Takahashi identity satisfied by $\Gamma$ :

$$
\int\left(t \nabla_{i}\left\langle V_{l}\right\rangle_{\bullet} \frac{\delta \Gamma}{\delta\left\langle V_{l}\right\rangle} \bullet t \nabla_{i}\left\langle\sigma_{l}\right\rangle_{\bullet} \frac{\delta \Gamma}{\delta\left\langle\sigma_{l}\right\rangle} \bullet-\frac{\delta \Gamma}{\delta\left\langle V_{i}\right\rangle}\right)=0 .
$$

The great utility of the WT identity Eq. (8) is that it provides exact, non-perturbative equations relating various proper vertices (correlation functions) associated with the NSE equation. At lowest order (i.e., tree level), these proper vertices can be read off directly from the action $S$ (see below). Here, and in conformity with the Reynolds decomposition, the mean value of the velocity field in the absence of source terms is a constant (and nonzero) vector: $\left\langle V_{i}(\mathbf{x}, t)\right\rangle=\delta W[\mathbf{J}, \mathbf{\Sigma}] /\left.\delta J_{i}\right|_{J=\Sigma=0}=K_{i}$. A nonzero expectation value for the fluid velocity in the limit of vanishing external sources is reminiscent of symmetry breaking in field theory. Indeed, the existence of a constant $K_{i}$ breaks the fluid isotropy since a preferred direction is being singled out. In this case, the correct Taylor series 
representation of the effective action $\Gamma$ is given by 14$]$

$$
\left(\left\langle V_{i m 1}(m 1)\right\rangle_{\bullet}-K_{i m 1}\right)\left\langle\sigma_{j 1}(1)\right\rangle_{\bullet} \ldots\left\langle\sigma_{j m 2}(m 2)\right\rangle_{\bullet}
$$

$$
\begin{gathered}
\Gamma\left[\langle\mathbf{V}\rangle_{\bullet},\langle\sigma\rangle_{\bullet}\right]=\sum_{m 1, m 2=1}^{\infty} \frac{1}{m 1 ! m 2 !} \int[d \mathbf{x} d t] \\
\Gamma_{i 1, i 2, \ldots, i m 1, j 1, j 2, \ldots, j m 2}^{(m 1, m 2)}\left(\left\langle V_{i 1}(1)\right\rangle_{\bullet}-K_{i 1}\right) \ldots \times
\end{gathered}
$$

where

$$
\Gamma_{i 1, i 2, \ldots, i m 1, j 1, j 2, \ldots, j m 2}^{(m 1, m 2)}=\left.\frac{\delta^{m 1+m 2} \Gamma\left[\langle\mathbf{V}\rangle_{\bullet},\langle\sigma\rangle_{\bullet}\right]}{\delta\left\langle V_{i 1}(1)\right\rangle_{\bullet} \ldots \delta\left\langle V_{i m 1}(m 1)\right\rangle_{\bullet} \delta\left\langle\sigma_{j 1}(1)\right\rangle_{\bullet} \ldots \delta\left\langle\sigma_{j m 2}(m 2)\right\rangle_{\bullet}}\right|_{\substack{\langle V\rangle_{\bullet}=K \\\langle\sigma\rangle_{\bullet}=0}}
$$

We employ a condensed notation to indicate the dependence on space and time, thus, e.g., $(1) \equiv\left(\mathbf{x}_{1}, t_{1}\right)$, $(m 1) \equiv\left(\mathbf{x}_{m 1}, t_{m 1}\right)$ and $[d \mathbf{x} d t]$ stands for the volume element for all $m 1+m 2$ pairs of space and time points to be integrated over in each term of the sum in Eq. (9). The factors Eq. (10) for each $m 1$ and $m 2$, correspond to specific proper vertex with $m 1$ factors of the velocity and $m 2$ factors of the conjugate field. So, for example, the inverse response function and proper vertex associated with the nonlinear convective term in the NSE are (suppressing the dependence on the space and time arguments) $\Gamma_{i j}^{(1,1)}$, and $\Gamma_{i j k}^{(2,1)}$, respectively.

The pertinent WT identity that we seek is obtained by inserting Eq. (9) into Eq. (8) differentiating the latter with respect to $\frac{\delta}{\delta\left\langle V_{k}\left(\mathbf{y}, t^{\prime}\right)\right\rangle_{\bullet}} \frac{\delta}{\delta\left\langle\sigma_{j}\left(\mathbf{w}, t^{\prime \prime}\right)\right\rangle_{\bullet}}$, using the definition in Eq. (10) and then setting $\langle V\rangle_{\bullet}=K$ and $\langle\sigma\rangle_{\bullet}=0$. Doing so yields

$$
\begin{aligned}
\left(t^{\prime \prime}\right. & \left.-t^{\prime}\right) \frac{\partial}{\partial y_{i}} \Gamma_{j k}^{(1,1)}\left(\mathbf{y}, t^{\prime}, \mathbf{w}, t^{\prime \prime}\right) \\
& =\int d \mathbf{x} d t \Gamma_{i j k}^{(2,1)}\left(\mathbf{x}, t, \mathbf{y}, t^{\prime}, \mathbf{w}, t^{\prime \prime}\right),
\end{aligned}
$$

which follows after an integration by parts (we adopt vanishing boundary conditions) and using the fact that spatial homogeneity implies proper vertices are translational invariant in configuration space. For our final step, we express this identity in terms of wavenumber (or, momentum) and frequency space $(\mathbf{k}, \omega)$, by means of the Fourier transform (FT). Recall that for stationary flow, the proper vertices can depend only on time differences. Thus, the FT of Eq. (11) yields

$$
-k_{i} \frac{\partial}{\partial \omega} \Gamma_{j k}^{(1,1)}(\mathbf{k}, \omega ;-\mathbf{k},-\omega)=\Gamma_{i j k}^{(2,1)}(\mathbf{0}, 0 ; \mathbf{k}, \omega ;-\mathbf{k},-\omega) .
$$

At zero-loop order (indicated via the zero superscript), and from Eq. (3) we can read off directly the 1PI functions that are related by this WT identity, namely

$$
\begin{aligned}
& \stackrel{0}{\Gamma}_{i j}^{(1,1)}=\left(-i \omega+i K_{j} k_{j}+\nu_{0} k^{2}\right) P_{i j}(\mathbf{k}) \\
& \stackrel{0}{\Gamma}_{i j k}^{(2,1)}=i \lambda_{0} k_{i} P_{j k}(\mathbf{k})
\end{aligned}
$$

It is important to realize that the $K_{j}$-dependence of the bare response function (13) follows from applying the Reynolds decomposition from the outset to the NSE, which leads to (11) and (3). This expression cannot be obtained from applying a Galilean transformation to the response function for an isotropic fluid $\left(K_{j}=0\right)$. Inserting Eqs. (13 14) into Eq. (12) implies that $\lambda_{0}=1$. In other words, Galilean invariance requires that the bookkeeping parameter introduced in Eq. (1) must be identically unity. This result is clearly independent of the mean velocity $K_{i}$. To go further, we make the reasonable assumption that some of the perturbative corrections to the solution of Eq. (1) can be absorbed into redefinitions of the parameters appearing in the equation of motion. This is to say, we assume the NSE is partially form-invariant with respect to the renormalization arising from the fluctuations inherent in the randomly stirred ensemble. This renormalization leads to frequency and wavevector dependent viscosity and a "mass" term $\Sigma$ in the inverse response function. The vertex associated with the convective term in the NSE can also in principle be modified or suffer renormalization, leading to a frequency and wavevector dependent term which we denote $\Lambda$. The most general terms one can write down which maintain the same tensorial structure as their unrenormalized counterparts Eq. (13) and Eq. (14) are

$$
\begin{aligned}
& \Gamma_{j k}^{(1,1)}(\mathbf{k}, \omega ;-\mathbf{k},-\omega)= \\
& \quad\left(-i \omega+\nu(\omega, k) k^{2}+\Sigma(\omega, k)\right) P_{j k}(\mathbf{k}) \\
& \Gamma_{i j k}^{(2,1)}(\mathbf{0}, 0 ; \mathbf{k}, \omega ;-\mathbf{k},-\omega)=i k_{i} P_{j k}(\mathbf{k})+\Lambda_{i j k}(\omega, \mathbf{k})
\end{aligned}
$$

where in accordance with Eq. (12) and Eqs. (1314), we have set $\lambda_{0}=1$ in Eq. (16). The relation of Eq. (15) and Eq. (16) to Eq. (13) and Eq. (14), respectively, is as follows. Imagine setting the nonlinear term in the NSE to zero by formally taking $\lambda_{0} \rightarrow 0$. Then $\nu(\omega, k) \rightarrow \nu_{0}$, $\Sigma(\omega, k) \rightarrow i K_{j} k_{j}$ and $\Lambda_{i j k}(\omega, k) \rightarrow 0$. That is, these terms appear as a result of the perturbation expansion, which is developed in powers of $\lambda_{0}$. Finally, inserting these expressions Eqs. (1516) into the Ward-Takahashi 
identity Eq. (12) implies that

$$
-k_{i} P_{j k}(\mathbf{k})\left(k^{2} \frac{\partial \nu(\omega, k)}{\partial \omega}+\frac{\partial \Sigma(\omega, k)}{\partial \omega}\right)=\Lambda_{i j k}(\omega, \mathbf{k})
$$

There are some general consequences one can draw from this identity. First of all, in models of stationary forced turbulence Eq. (1), the effective viscosity can not depend on time (nor will its FT depend on the frequency $\omega$ ), and thus at most the renormalized viscosity could depend on wavenumber: $\nu=\nu\left(k^{2}\right)$. The same holds for the "mass" term $\Sigma=\Sigma(k)$, so that for stationary random forcing Eq. (17) immediately implies that $\Lambda_{i j k}(\omega, \mathbf{k})=0$, and hence the vertex function $\Gamma_{i j k}^{(2,1)}$ in Eq. (16) is not altered by perturbative corrections to the equation of motion. When $\Lambda_{i j k}(\omega, \mathbf{k})=0$, this vertex is that which corresponds to the convective term in the NSE Eq. (1). There is one important caveat here: this conclusion holds only for the case of zero-momentum transfer in the vertex (see Eq. (12)). We are not claiming that the vertex cannot have nontrivial renormalization for finite momentum transfer. This result of vanishing vertex correction $\Lambda_{i j k}(\omega, \mathbf{k})$ is valid only for large-scale flows and is independent of the value of $K_{i}$.

In conclusion, when we come to reconcile Galilean invariance with fluctuation phenomena, we know that the enforcement of this symmetry does impose nontrivial conditions on some of the physical parameters appearing in the equation of motion. For example, recursive renormalization group (RG) procedures applied to the NSE explicitly generate higher-order nonlinearities in the renormalized fluid equation of motion. These higher-order nonlinearities, such as triple velocity products, do however preserve Galilean invariance [15]. Correlation functions provide further information that can not be ascertained at the level of the evolution equation of motion alone. Here it is worthwhile to recall that the original operator-based perturbation theory developed by MSR requires three different vertices. They claim that the whole problem of strong turbulence is contained in a proper treatment of the vertex renormalization [12]. The two additional vertices introduced by MSR have no counterpart at the level of the NSE.

In contrast to the NSE, correlation functions can not be neatly separated into mean and fluctuating parts. This in general means constraints from Galilean invariance for the mean velocity component also can have implications for the fluctuating component. In particular, this leads to the WT identity Eq. (17), which provides a nontrivial relation between the fluid response function and the nonlinear vertex term (convective term). This dependence holds of course only for the large scale flow regime and says nothing about the small scale flow behavior i.e., the inertial and dissipation ranges). This is so because (17) follows directly from (12), which as can be checked, involves the vertex only for zero momentum transfer.

In [], the claim is made that Galilean invariance provides no constraint whatsoever on the vertex renormalization, in sharp contrast to the results in [2, 3, 6]. These previous papers that examined the WT identities did so in the frame at zero mean velocity in which it would be impossible to test the claims of [7], which depend crucially on having Reynolds decomposed the fluid velocity. To test these claims requires working in a frame of general nonzero mean velocity. This paper has done this and demonstrated that although the claims in [7] have some validity, the fluctuating velocity component is still partly constrained, in particular in the limit of zero momentum transfer. Thus our result provides a resolution to the seemingly differing conclusions drawn in 7] and in [2, 3, 6]. In the process of addressing this issue, our paper has also provided the most general form of the WT identities for the randomly forced NSE. These results can not be derived from the other treatments of the WT identities in 2, 3, 6] by simply performing a Galilean transformation of their results. Further understanding about the implications of Galilean invariance in the NSE can perhaps be gained by examining the analogy between this symmetry and global gauge invariance in QFT and exploring the implications of gauge fixing procedures.

We thank R. Horsley and W.D. McComb for helpful discussions. A.B. was funded by the U.K. Particle Physics and Astronomy Research Council (PPARC). D.H. acknowledges a U.K. Royal Society funded Study Visit and the CSIC "Marina Bueno" program.
[1] S.B. Pope, Turbulent Flows (Cambridge University Press, Cambridge, 2000).

[2] D. Forster, D.R. Nelson and M.J. Stephen, Phys. Rev. A16, 732 (1977).

[3] C. DeDominicis and P.C. Martin, Phys. Rev. A19, 419 (1979).

[4] E.V. Teodorovich, J. Applied Math. and Mech. 53, 340 (1989).

[5] C-H. Mou and P.B. Weichmann, Phys. Rev. E52, 3738 (1995).

[6] R. Collina and P. Tomassini, hep-th/9709185v2, (1997).

[7] W.D. McComb, Phys. Rev. E71, 037301 (2005).
[8] C. DeDominicis, J. Phys. (Paris) Colloq. 1, 247 (1976).

[9] H.K. Janssen, Z. Phys. B 23, 377 (1976).

[10] R. Phythian, J. Phys. A 10, 777 (1977).

[11] R.D. Jensen, J. Stat. Phys. 25, 183 (1981).

[12] P.C. Martin, E.D. Siggia and H.A. Rose, Phys. Rev. A8, 423 (1973).

[13] J. Zinn-Justin, Quantum Field Theory and Critical Phenomena (Oxford University Press, Oxford, 2002) 4rth ed.

[14] E.S. Abers and B.W. Lee, Phys. Lett. 9C, 1-141 (1973).

[15] Y. Zhou and G. Vahala, Phys. Rev. E 48, 4387 (1993). 